\title{
Alongamento muscular na dor lombar crônica inespecífica: uma estratégia do método GDS
}

\author{
Stretching in nonspecific chronic low back pain: a strategy of the GDS method
}

Maria Angélica Ferreira Leal Puppin ${ }^{1}$, Amélia Pasqual Marques ${ }^{2}$, Ary Gomes da Silva 3 , Henrique de Azevedo Futuro Neto ${ }^{4}$

Estudo desenvolvido na Clínica de Fisioterapia do UVV - Centro Universitário Vila Velha - Vila Velha (ES), Brasil.

${ }^{1}$ Doutora; Professora do Curso de Fisioterapia do Centro Universitário Vila Velha (UVV) Vila Velha (ES), Brasil.

${ }^{2}$ Doutora; Professora do Programa de Pós-Graduação em Ciências da Reabilitação da Faculdade de Medicina da Universidade de São Paulo (FOFITO/FMUSP) - São Paulo (SP), Brasil.

${ }^{3}$ Doutor; Professor Doutor da UVV - Vila Velha (ES), Brasil.

${ }^{4}$ Doutor; Professor da Escola Superior de Ciências da Santa Casa de Misericórdia de Vitória (EMESCAM) e da UNIVIX Vitória (ES), Brasil.

\section{ENDEREÇO PARA CORRESPONDÊNCIA}

Maria Angélica Ferreira Leal Puppin - Rua Mercúrio s/n CEP: 29102-800 - Vila Velha (ES), Brasil - E-mail: angelica. puppin@uvv.br

APRESENTACÃO: abr. 2010

ACEITO PARA PUBLICAÇÃO: jan. 2011

FONTE DE FINANCIAMENTO: nenhuma

CONFLITO DE INTERESSES: nada a declarar
RESUMO: O objetivo deste estudo foi verificar a eficácia do alongamento muscular, usando uma sequência proposta pelo Método Godelieve Denys-Struyf (GDS) na redução da dor, na incapacidade funcional, no aumento da flexibilidade global e na capacidade de contração do músculo transverso do abdome (TrA), em indivíduos com dor lombar crônica inespecífica. Participaram 55 pacientes, de 18 a 60 anos, divididos em dois grupos: Grupo Alongamento $(n=30)$ submetido a exercícios de alongamento, duas vezes por semana, e Grupo Controle $(n=25)$ que não realizou tratamento. A dor foi avaliada pela escala visual analógica; a incapacidade funcional, pelo Índice de Oswestry; a flexibilidade global, pelo terceiro dedo ao solo; e a capacidade de contração do TrA, pela unidade de biofeedback pressórico. Foram realizadas três avaliações, inicial, após 8 e 16 semanas da inicial. Foi considerado nível de significância de $\alpha<0,05$. Os resultados mostram que o Grupo Alongamento apresentou diminuição na dor, incapacidade funcional e aumentou a flexibilidade global $(p<0,001)$ após 8 e 16 semanas $(p<0,05)$, porém não melhorou a capacidade de contração do $\operatorname{Tr} A(p=0,13)$. A sequência de alongamentos usada no método GDS mostra-se eficaz na redução da dor, incapacidade funcional e melhora da flexibilidade global em pacientes com dor lombar crônica inespecífica.

Descritores: dor lombar; exercícios de alongamento muscular; fisioterapia.

ABSTRACT: The purpose of this study was to assess the effectiveness of stretching exercises of the Muscle and Joint Chains Godelieve Denys-Struyf (GDS) method in pain reduction, functional disability, increase global flexibility, and ability of the transversus abdominis muscle $(\operatorname{TrA})$ contraction in individuals with nonspecific chronic low back pain. Fifty-five patients, from 18 to 60 years-old, participated in this study divided into two groups: the Stretching Group $(n=30)$ underwent stretching exercises twice a week; the Control Group $(n=25)$ was subjected only to evaluation. Pain was assessed by a visual analogue scale, functional disability by the Oswestry Questionnaire, global flexibility by third finger to the ground test and the ability of TrA contraction by a pressure biofeedback unit. Three evaluations were performed, starting after 8 and 16 weeks from the beginning. Significance level was $\alpha<0.05$. Results show that the Stretching Group presented a decrease in pain, functional disability, and increased global flexibility $(p<0.001)$ after 8 and 16 weeks $(p<0.05)$. No improvement in the ability of TrA muscle contraction was demonstrated $(p=0.13)$. The sequence of stretching exercises used in the GDS method is effective in reducing pain, functional disability, and improving global flexibility in patients with nonspecific chronic low back pain.

KeYwords: low back pain; muscle stretching exercises; physical therapy. 


\section{INTRODUCÃO}

A lombalgia crônica é definida como a dor localizada entre a margem costal e a prega glútea inferior, com ou sem irradiação para membros inferiores, que persiste por pelo menos 12 semanas ${ }^{1}$. Um diagnóstico específico não é feito em $80 \%$ dos casos, permanecendo baseado na localização da dor e na sua duração ${ }^{2}$. Aproximadamente $90 \%$ dos casos têm resolução espontânea em seis semanas, sendo que de 2 a $7 \%$ se tornam crônicos ${ }^{3}$. A cronicidade e a incapacidade funcional são problemas relacionados à lombalgia e, apesar do grande investimento no tratamento, $\mathrm{O}$ sucesso permanece baixo ${ }^{4}$.

Na Fisioterapia, a cinesioterapia é a primeira linha de escolha ${ }^{1,5,6}$, sendo o alongamento, especialmente o estático ${ }^{7,8}$, muito utilizado. Rosário, Marques e Maluf ${ }^{9}$ relataram ser de 30 segundos o tempo ideal de alongamento para músculos de adultos saudáveis.

O método de cadeias musculares e articulares Godelieve Denys-Struyf (GDS) utiliza o alongamento estático como uma das estratégias de tratamento da lombalgia, avalia a cadeia muscular relacionada à dor e utiliza massagens, estabilização lombar e conscientização corporal, além do alongamento, para restabelecer o equilíbrio dos músculos e articulações da região lombopélvica ${ }^{10,11}$. Em relação ao alongamento, propõe-se o início pelas cadeias posteriores para melhorar a flexibilidade dos músculos rotadores externos do quadril; a seguir, alongam-se as cadeias anteriores, principalmente os rotadores internos, flexores e adutores do quadril; por último, são alongados os músculos extensores do quadril, flexores do joelho e da perna e das cadeias posteriores. Para GDS, a flexibilidade do quadril melhora a postura lombopélvica e protege a coluna lombar de alterações biomecânicas ${ }^{10}$.

Diretrizes internacionais ${ }^{1}$ sugerem a realização de pesquisas para analisar as especificidades dos exercícios usados na lombalgia, assim, este estudo tem como objetivo avaliar o efeito do alongamento, utilizando uma sequência de exercícios definida pelo método GDS na dor, incapacidade funcional, flexibilidade global e capacidade de contração do músculo transverso do abdome (TrA), em pacientes com dor lombar crônica inespecífica.

\section{METODOLOGIA}

Foram recrutados 62 indivíduos com lombalgia na Clínica de Fisioterapia do Centro Universitário Vila Velha (UVV), no Espírito Santo. Sete deles abandonaram o tratamento e 55 foram divididos aleatoriamente em dois grupos: Grupo Alongamento $(n=30)$ com 15 homens e 15 mulheres, submetidos a alongamentos, e Grupo Controle $(n=25)$ composto por 15 mulheres e 10 homens, nãotratados. No sorteio, o primeiro paciente participaria do Grupo Alongamento, o segundo do Controle e, assim, sucessivamente, conforme ordem de chegada à clínica.

Foram incluídos pacientes com diagnóstico clínico de lombalgia inespecífica crônica, que tinham entre 18 e 60 anos e foram excluídos os que tinham doenças cardiovasculares descompensadas; distúrbios psiquiátricos; doenças infectocontagiosas; disfunções neurológicas; tumor ou cirurgia na lombar, pelve e membros inferiores; artrose de quadril; mulheres grávidas ou no puerpério; índice de massa corporal - IMC $>30$; pacientes realizando Fisioterapia e os que não realizaram todas as sessões de fisioterapia e avaliações.

O cálculo amostral considerou $80 \%$ de poder estatístico, desvio padrão de dois pontos, melhora minimamente significante de $20 \%$ e nível de significância de $5 \%$.

O estudo foi aprovado pelo Comitê de Ética e Pesquisa da UVV, registro $\mathrm{n}^{\circ}$ $112 / 2008$ e todos os pacientes assinaram um termo de consentimento livre e esclarecido.

\section{Procedimentos e intervenção}

Os participantes realizaram três avaliações: inicial (A1), após oito semanas (A2) e após 16 semanas da inicial (A3), por uma única pesquisadora. No Grupo Alongamento, A1 correspondeu à avaliação pré-tratamento; A2 ao póstratamento e A3 a oito semanas após término do tratamento.
A dor foi avaliada pela escala visual analógica $^{12,13}$, uma escala de $10 \mathrm{~cm}$ na qual os pacientes assinalavam seu nível álgico. Valores de 0 a 3 correspondem à dor leve, 4 a 7 à moderada e 8 a 10 à intensa.

A incapacidade funcional foi avaliada pelo Índice de Incapacidade de Oswestry ${ }^{14}$, validado para língua portuguesa $^{15}$, sendo a interpretação realizada em porcentagem: 0 a 20\%, incapacidade mínima; 21 a 40\%, incapacidade moderada; 41 a $60 \%$, incapacidade severa; 61 a $80 \%$, invalidez; e 81 a 100\%, paciente acamado ou exagera nos sintomas ${ }^{16}$.

A flexibilidade global foi avaliada pelo teste do terceiro dedo ao solo. Os pacientes realizavam uma flexão anterior do tronco, na tentativa de tocar o solo com os dedos e media-se a distância, com fita métrica flexível ${ }^{17,18}$.

A capacidade de contração do TrA foi verificada pela unidade de biofeedback pressórica (UBP), marca Chantanooga, um transdutor pressórico com bolsa inflável não-distensível, um catéter e um esfigmomanômetro. A habilidade de deprimir o abdome contra a coluna lombar diminui a pressão na bolsa, que é registrada pelo esfigmomanômetro. A redução pressórica de 4 a $10 \mathrm{mmHg}$, a partir de $70 \mathrm{mmHg}$, é considerada como ótima contração do $\operatorname{Tr} \mathrm{A}^{19}$.

A UBP é um método de baixo custo, não-invasivo e de fácil utilização ${ }^{20}$. Durante a medida, a bolsa foi inflada a $70 \mathrm{mmHg}$ e posicionada entre as espinhas ilíacas anterossuperiores e a cicatriz umbilical, com o paciente em decúbito ventral. Foi solicitada a contração do TrA, levando o abdome em direção à coluna, sem movimentos lombopélvicos, mantendo respiração calma. Três contrações foram realizadas durante a expiração, mantidas por 10 segundos e o valor médio foi registrado.

Os pacientes do Grupo Alongamento foram submetidos a 16 sessões de 40 minutos, duas vezes por semana. Realizaram seis tipos de alongamento, durante 30 segundos e mesmo tempo de descanso, os exercícios foram repetidos três vezes em cada membro e foram acompanhados por uma fisioterapeuta com formação no Método GDS. 
Os dois primeiros exercícios foram realizados para alongar músculos rotadores externos e extensores do quadril das cadeias posterolaterais; o terceiro foi para flexores e rotadores internos do quadril, das cadeias anteroposteriores e anterolaterais; o quarto para adutores do quadril, das cadeias anteromedianas e os dois últimos para extensores do quadril, joelho e perna, das cadeias posteromedianas.

O Grupo Controle não foi tratado, somente avaliado. Após A3, os pacientes foram encaminhados à Fisioterapia da Clínica da UVV.

\section{Análise estatística}

Verificou-se a normalidade dos dados pelo teste Kolmogorov-Smirnov. Os grupos foram analisados nas avaliações A1, A2 e A3. Utilizou-se o teste $t$ de Student para dados antropométricos e clínicos. A dor, incapacidade, flexibilidade e capacidade de contração do TrA foram submetidas aos testes de Friedman da ANOVA de fator único, com medidas repetidas e Tukey na análise intragrupo e à ANOVA de fator único com medidas repetidas e teste de Holm-Sidak na análise entre grupos. Utilizou-se o programa SigmaStat, versão 3.5, e considerou-se o nível de significância $\alpha<0,05$.

\section{RESULTADOS}

Características antropométricas e clínicas dos grupos são mostradas na Tabela 1. Não houve diferença significante em nenhuma das variáveis $(p>0,05)$. No Grupo Controle, $60 \%$ dos indivíduos eram mulheres e, no Grupo Alongamento, a distribuição foi homogênea (50\%).
Houve $11 \%$ de desistência: $6,2 \%$ no Alongamento e $4,8 \%$ no Controle. A principal causa foi retorno ao trabalho, após licença-saúde. Não houve diferença significante nas variáveis estudadas entre o Grupo Alongamento e os pacientes desistentes.

A Tabela 2 apresenta dados dos grupos estudados, em três avaliações. O Grupo Alongamento mostrou diferença significante na dor, incapacidade e flexibilidade $(p<0,001)$ entre as três avaliações, o mesmo foi observado entre A1 e A2 e A1 e A3 $(p<0,05)$. Não houve diferença entre A2 e A3 ( $p>0,05)$, mostrando que os indivíduos mantiveram os ganhos após oito semanas. Não houve diferença na capacidade de contração do TrA nas três avaliações $(p=0,13)$. O Grupo Controle não apresentou diferenças entre A1, A2 e A3 em nenhuma das variáveis.

A Tabela 3 mostra a comparação entre os grupos estudados. Houve diferença significante entre os grupos na dor, incapacidade e flexibilidade $(p<0,001)$, não houve diferença na capacidade de contração do $\operatorname{TrA}(p=0,07)$. Nos testes post hoc, houve diferença significante entre: A2 do Grupo Alongamento e os três momentos do Grupo Controle $(p<0,05)$ na dor, incapacidade e flexibilidade, o mesmo foi encontrado entre A3 do Grupo Alongamento e os momentos A1, A2 e A3, do Grupo Controle. Não houve diferença entre A3 do Grupo Alongamento e A2 do Controle $(p=0,10)$ na flexibilidade global.

\section{DISCUSSÃO}

Os exercícios para lombalgia são divulgados e recomendados ${ }^{1}$. Contudo, são raros estudos que utilizam técnicas isoladas para comparar o efeito do tratamento com um Grupo Controle nãotratado ${ }^{11,21-23}$. Este estudo comparou o efeito do alongamento, de forma isolada, utilizando uma sequência de exercícios definida pelo método GDS, com um Grupo Controle não-tratado, na dor lombar crônica inespecífica. Encontrou-se redução da dor, incapacidade funcional e aumento da flexibilidade global somente no Grupo Alongamento. Não foi encontrada melhora na capacidade de contração do TrA.

Os presentes resultados mostraram que a dor diminuiu de moderada para leve e a incapacidade, de moderada para mínima, após o tratamento com alongamentos. Os ganhos obtidos se mantiveram depois de oito semanas. A redução da dor e, consequentemente da incapacidade, foram atribuídas à melhora da flexibilidade global. Músculos flexíveis diminuem o estresse compressivo articular, melhoram a postura e mobilidade lombopélvica e do quadril, facilitando o reaprendizado motor funcional desta região ${ }^{9,10,24}$. Resultados semelhantes foram encontrados por Díaz Arribas et al. ${ }^{11}$, os quais compararam o método GDS com eletrotermoterapia na lombalgia inespecífica, verificando redução da dor e incapacidade no Grupo GDS. Porém, neste estudo, os autores associam diversas técnicas de tratamento, inclusive alongamento, no Grupo GDS, não esclarecendo o efeito de cada modalidade no tratamento da lombalgia.

Em relação à flexibilidade global, somente no Grupo Alongamento foram encontrados ganhos após o tratamento e depois de oito semanas. Resultados

Tabela 1. Dados antropométricos e clínicos dos Grupos Alongamento e Controle na avaliação inicial (A1)

\begin{tabular}{lccc}
\hline Variáveis & $\begin{array}{c}\text { Grupo Alongamento }(\mathrm{n}=30) \\
\text { Média/DP }\end{array}$ & $\begin{array}{c}\text { Grupo Controle }(\mathrm{n}=25) \\
\text { Média/DP }\end{array}$ & Valor $\mathrm{p}^{*}$ \\
\hline Idade (anos) & $37,5(12,1)$ & $37,8(13,6)$ & 0,94 \\
Índice de massa corpórea $\left(\mathrm{kg} / \mathrm{m}^{2}\right)$ & $25,1(2,9)$ & $24,2(2,2)$ & \\
Sexo $(\%)$ & & & \\
Feminino & $15(50 \%)$ & $15(60 \%)$ & \\
Masculino & $15(50 \%)$ & $125,5(16,9)$ & 0,51 \\
Pressão sistólica $(\mathrm{mmHg})$ & $128,4(16,3)$ & $78,7(10,7)$ & 0,84 \\
Pressão diastólica $(\mathrm{mmHg})$ & $79,2(8,1)$ & $76,0(11,8)$ & 0,72 \\
Frequência cardíaca $(\mathrm{bpm})$ & $77,4(15,2)$ & & \\
\hline
\end{tabular}

* teste $t$ de Student para $\alpha=0,05$ 
Tabela 2. Análise das variáveis estudadas nos pacientes dos Grupos Alongamento e Controle, na avaliação inicial (A1), após oito (A2) e 16 semanas (A3)

\begin{tabular}{|c|c|c|c|c|c|c|c|c|}
\hline \multirow{3}{*}{ Variáveis } & \multicolumn{3}{|c|}{ Grupo Alongamento $(n=30)$} & \multirow{3}{*}{ Valor $p$} & \multicolumn{3}{|c|}{ Grupo Controle $(n=25)$} & \multirow{3}{*}{ Valor $p$} \\
\hline & A1 & A2 & A3 & & A1 & A2 & A3 & \\
\hline & \multicolumn{3}{|c|}{ Mediana $[25 \% ; 75 \%]$} & & \multicolumn{3}{|c|}{ Mediana [25\%; 75\%] } & \\
\hline \multirow[t]{6}{*}{ Dor } & 4,0 & 1,0 & 1,5 & & 5,0 & 4,0 & 5,0 & \\
\hline & {$[3,0 ; 6,0]$} & {$[0,0 ; 3,0]$} & {$[0,0 ; 5,0]$} & $<0,001^{*}$ & {$[3,0 ; 7,0]$} & {$[1,7 ; 6,0]$} & {$[2,0 ; 6,2]$} & $0,36^{*}$ \\
\hline & 4,0 & 1,0 & & & & & & \\
\hline & {$[3,0 ; 6,0]$} & {$[0,0 ; 3,0]$} & & $<0,05^{* *}$ & & & & \\
\hline & 4,0 & & 1,5 & & & & & \\
\hline & {$[3,0 ; 6,0]$} & & {$[0,0 ; 5,0]$} & $<0,05$ & & & & \\
\hline \multirow[t]{6}{*}{ Incapacidade } & 26,0 & 11,0 & 18,0 & & 24,0 & 22,0 & 22,0 & \\
\hline & {$[18,0 ; 34,0]$} & {$[4,0 ; 22,0]$} & {$[8,9 ; 26,0]$} & $<0,001^{*}$ & {$[18,0 ; 36,5]$} & {$[14,0 ; 36,4]$} & {$[13,5 ; 36,4]$} & $0,20^{*}$ \\
\hline & 26,0 & 11,0 & & & & & & \\
\hline & {$[18,0 ; 34,0]$} & {$[4,0 ; 22,0]$} & & $<0,05$ & & & & \\
\hline & 26,0 & & 18,0 & & & & & \\
\hline & {$[18,0 ; 34,0]$} & & {$[8,9 ; 26,0]$} & $<0,05^{* *}$ & & & & \\
\hline \multirow[t]{6}{*}{ Flexibilidade } & 16,5 & 0,0 & 4,0 & & 10,0 & 9,0 & 9,0 & \\
\hline & {$[12,0 ; 24,0]$} & {$[0,0 ; 7,0]$} & {$[0,0 ; 12,0]$} & $<0,001^{*}$ & {$[0,0 ; 25,2]$} & {$[0,0 ; 21,0]$} & {$[0,0 ; 22,7]$} & $0,36^{*}$ \\
\hline & 16,5 & 0,0 & & & & & & \\
\hline & {$[12,0 ; 24,0]$} & {$[0,0 ; 7,0]$} & & $<0,05^{* *}$ & & & & \\
\hline & 16,5 & & 4,0 & & & & & \\
\hline & {$[12,0 ; 24,0]$} & & {$[0,0 ; 12,0]$} & $<0,05^{* *}$ & & & & \\
\hline Contração & $-4,0$ & $-4,5$ & $-4,0$ & & $-4,0$ & $-4,0$ & $-4,0$ & \\
\hline do $\operatorname{Tr} A$ & {$[-5,0 ;-2,0]$} & {$[-6,0 ;-4,0]$} & {$[-6,0 ;-3,0]$} & $0,13 *$ & {$[-6,0 ;-1,5]$} & {$[-6,0 ;-2,0]$} & {$[-6,0 ;-2,0]$} & $0,83^{*}$ \\
\hline
\end{tabular}

*teste de Friedman da ANOVA fator único com medidas repetidas; ** teste de Tukey para $\alpha=0,05$

Tabela 3. Comparação dos Grupos Alongamento e Controle na avaliação inicial (A1), após oito (A2) e 16 semanas (A3) em relação às variáveis estudadas

\begin{tabular}{|c|c|c|c|}
\hline \multirow[t]{2}{*}{ Variável } & $\begin{array}{c}\text { Grupo Alongamento } \\
(\mathrm{n}=30)\end{array}$ & $\begin{array}{c}\text { Grupo Controle } \\
(\mathrm{n}=25)\end{array}$ & \multirow[b]{2}{*}{ Valor $\mathrm{p}$} \\
\hline & \multicolumn{2}{|c|}{ Média/DP } & \\
\hline \multicolumn{4}{|l|}{ Dor } \\
\hline A1 & $4,4(2,7)$ & $4,9(2,5)^{12}$ & 0,32 \\
\hline $\mathrm{A} 2$ & $1,5(1,6)^{1}$ & $3,8(2,4)^{12}$ & 0,000 \\
\hline A3 & $2,4(2,7)^{2}$ & $4,1(2,7)^{12}$ & 0,003 \\
\hline \multicolumn{4}{|c|}{ Incapacidade } \\
\hline A1 & $28,1(12,3)$ & $27,8(13,7)^{12}$ & 0,69 \\
\hline A2 & $13,6(12,3)^{1}$ & $25,1(14,2)^{12}$ & 0,000 \\
\hline A3 & $18,4(13,3)^{2}$ & $25,5\left(15,50^{12}\right.$ & 0,009 \\
\hline \multicolumn{4}{|c|}{ Flexibilidade } \\
\hline A1 & $17,2(9,2)$ & $13,1(13,4)^{12}$ & 0,08 \\
\hline $\mathrm{A} 2$ & $3,9(5,8)^{1}$ & $11,4(12,6)^{1}$ & 0,004 \\
\hline A3 & $7,0(8,5)^{2}$ & $12,8(14,0)^{12}$ & 0,03 \\
\hline
\end{tabular}

* ANOVA de fator único com medidas repetidas para $\alpha=0,05$. Os números ${ }^{12}$ identificam diferenças significantes no teste de Holm-Sidak de comparação entre grupos, em pares de diferentes procedimentos para $\alpha=0,05$ semelhantes foram encontrados, por Sugano e Nomura ${ }^{25}$, em pacientes com lombalgia tratados com alongamento. A melhora da flexibilidade pode ser atribuída ao aumento no número de sarcômeros em série na fibra muscular e melhora nas propriedades viscoelásticas musculotendíneas ${ }^{26,27}$. Hiperatividade dos músculos superficiais do tron $\mathrm{CO}^{28} \mathrm{e}$ alterações na ativação muscular, especialmente cocontração dos agonistas e antagonistas do quadril ${ }^{29}$, podem ocorrer na lombalgia crônica, causando rigidez, limitação de movimentos e cargas compressivas na região lombopélvica, que são fatores de risco para degeneração e dor $^{30-32}$. Assim, técnicas que melhoram a flexibilidade, como a utilizada neste estudo, podem diminuir a dor e a incapacidade funcional.

Após o término do tratamento, os resultados obtidos tiveram pequenas perdas em relação à dor, incapacidade 
funcional e flexibilidade, o que leva os autores a sugerirem que o alongamento deve ser incorporado ao cotidiano dos pacientes.

Não encontrou-se melhora na capacidade de contração do TrA nos grupos estudados, verificando que o alongamento não alterara efetivamente a atividade motora deste músculo. Uma vez que pacientes com lombalgia crônica podem apresentar hipoatividade dos músculos profundos do tronco, entre esses, o TrA, gerando instabilidade lombar e alterações posturais $^{33,34}$, sugere-se a associação de atividades específicas para recrutamento dos músculos profundos do tronco aos alongamentos ${ }^{35-37}$.

Assim, o método das cadeias musculares e articulares GDS, ao associar técnicas de massagens, alongamentos, exercícios de estabilização, conscientização corporal e orientações para o cotidiano $^{24}$, no tratamento da lombalgia, provavelmente, contribui de forma efetiva para a reorganização das cadeias musculares e articulares do corpo.

Neste estudo, não foi avaliada a relação terapeuta e paciente, pois, no Grupo
Controle, não ocorreu intervenção ou relação terapêutica.

\section{CONCLUSÃO}

Os exercícios de alongamento, propostos pelo método de cadeias musculares e articulares GDS, foram eficazes na redução da dor, incapacidade funcional e no aumento da flexibilidade global, porém não melhoraram a capacidade de contração do músculo transverso do abdome em pacientes com dor lombar crônica inespecífica.

\section{REFERÊNCIAS}

1. Airaksinen O, Brox JI, Cedraschi C, Hildebrandt J, Klaber-Moffaett J, Kovacs F, et al. COST B13 Working Group on Guidelines for Chronic Low Back Pain: Chapter 4. European guidelines for the management of chronic nonspecific low back pain. Eur Spine J. 2006;2(suppl 15):S192-300.

2. Waddell G. Subgroups within "nonspecific" low back pain. J Rheumatol. 2005,32:395-6.

3. Manchikanti L, Singh V, Datta S, Cohen SP, Hirsch JA. Comprehensive review of epidemiology, scope, and impact of spinal pain. Pain Phys. 2009;12(4):E35-70.

4. Walker BF, Muller R, Grant WD. Low back pain in Australian adults: health provider utilization and care seeking. J Man Physiol Ther. 2004;27:327-35.

5. Hayden JA, Van Tulder MW, Malmivaara AV, Koes BW. Meta-analysis: exercise therapy for nonspecific low back pain. Ann Intern Med. 2005;142(9):765-75.

6. Chou R, Qaseem A, Snow V, Casey D, Cross Jr. JT, Shekelle $\mathrm{P}$, et al. Diagnosis and Treatment of Low Back Pain: A Joint Clinical Practice Guideline from the American College of Physicians and the American Pain Society. Ann Intern Med. 2007;147(7):479-91.

7. Davis DS, Ashby PE, McCale KL, McQuain JA, Wine JM. The effectiveness of 3 stretching techniques on hamstring flexibility using consistent stretching parameters. J Strength Cond Res. 2005;19(1):27-32.

8. Marek SM, Cramer JT, Fincher AL, Massey LL, Dangelmaier SM, Purkayastha S, et al. Acute Effects of Static and Proprioceptive Neuromuscular Facilitation Stretching on Muscle Strength and Power Output. J Athl Train. 2005;40(2):94-103.

9. Rosário JLP, Marques AP, Maluf AS. Aspectos Clínicos do alongamento: uma revisão da literatura. Rev Bras Fisioter. 2004;8:83-8.
10. Campignion P. Aspectos biomecânicos - cadeias musculares e articulares, método GDS - Noções básicas. São Paulo: Summus; 2003.

11. Díaz Arribas MJ, Ramos Sánchez M, Pardo Hervás $P$, Lópes Chicharro J, Ângulo Carreré T, et al. Effectiveness of the physical therapy Godelive Denys-Struyf method for nonspecific low back pain: primary care randomized control trial. Spine. 2009;34(15):1529-38.

12. Pengel LHM, Refshauge, KM, Maher CG. Responsiveness of Pain, Disability, and Physical Impairment Outcomes in Patients with Low Back Pain. Spine. 2004;29(8):879-83.

13. Mannion AF, Balagué F, Pellisé F, Cedraschi C. Pain measurement in patients with low back pain. Nat Clin Pract Rheumatol. 2007;3(11):610-8.

14. Fairbank JC. The use of revised Oswestry disability questionnaire. Spine. 2000;25(21):2846-7.

15. Vigato R, Alexandre NMC, Correa Filho HR. Development of a Brazilian Portuguese Version of the Oswestry Disability Index. Spine. 2007;32(4):481-6.

16. Coelho RA, Siqueira FB, Ferreira PH, Ferreira ML. Responsiveness of the Brazilian-Portuguese version of the Oswestry Disability Index in subjects with low back pain. Eur Spine J. 2008;17:1101-6.

17. Perret C, Poiraudeau S, Fermanian J, Colau MM, Benhamou MA, Revel M. Validity, reliability, and responsiveness of the fingertip-to-floor test. Arch Phys Med Rehabil. 2001;82:1566-70.

18. Marques AP. Manual de goniometria. 2a. ed. São Paulo: Editora Manole; 2003.

19. Anonymous. Stabilizer pressure bio-feedback: Operating Instructions. Brisbane: Chattanooga Pacific; 2002.

20. von Garnier K, Köveker K, Rackwitz B, Kober U, Wilke S, Ewert $T$, et al. Reliability of a test measuring transversus 


\section{Referências (cont.)}

abdominis muscle recruitment with a pressure biofeedback unit. Physiotherapy. 2009;95(1):8-14.

21. Häkkinen A, Ylinen J, Kautiainen $H$, Taryainen $U$, Kiviranta I. Effects of home strength training and stretching versus stretching alone after lumbar disk surgery: a randomized study with a 1-year follow-up. Arch Phys Med Rehabil. 2005;86(5):865-70.

22. UK BEAM Trial Team. United Kingdom back pain exercise and manipulation randomized trial: effectiveness of physical treatments for back pain in primary care. BMJ. 2005;329:1377-81.

23. O'Sullivan K, Murray E, Sainsbury D. The effect of warm-up, static stretching and dynamic stretching on hamstring flexibility in previously injured subjects. BMC Musculoskelet Disord. 2009;16:10-37.

24. Campignion P. Cadeias anterolaterais: cadeias musculares e articulares, método G.D.S. São Paulo: Summus; 2008.

25. Sugano A, Nomura T. Influence of water exercise and land stretching on salivary cortisol concentrations and anxiety in chronic low back pain patients. J Physiol Anthropol Appl Human Sci. 2000;19(4):175-80.

26. Ferreira GN, Teixeira-Salmela LF, Guimarães CQ. Gains in flexibility related to measures of muscular performance: impact of flexibility on muscular performance. Clin J Sport Med. 2007;17(4):276-81.

27. Coutinho EL, Gomes AR, França CN, Oishi J, Salvini TF. Effect of passive stretching on the immobilized soleus muscle fiber morphology. Braz J Med Biol Res. 2004;37(12):1853-61.

28. Hodges PW, Moseley GL. Pain and motor control of the lumbopelvic region: effect and possible mechanisms. J Electromyogr Kinesiol. 2003;13:361-70.
29. Nelson-Wong E, Gregory DE, Winter DA, Callaghan JP. Gluteus medius muscle activation patterns as a predicto of low back pain during standing. Clin Biomech. 2008;23(5):545-53.

30. MacDonald D, Moseley GL, Hodges PW. People with recurrent low back pain respond differently to trunk loading despite remission from symptoms. Spine. 2010.35(7):818-24.

31. Lamoth CJ, Meijer OD, Daffertshofer A, Wuisman PI, Beek PJ. Effects of chronic low back pain on trunk coordination and back muscle activity during walking: changes in motor control. Eur Spine J. 2006;15(1):23-40.

32. Cluas A, Hides J, Moseley GL, Hodges P. Sitting versus standing: does the intradiscal pressure cause disc degeneration or low back pain? J Electromyogr Kinesiol. 2008;18(4):550-8.

33. Arendt-Nielsen L, Graven-Nielsen T. Muscle pain: sensory implications and interaction with motor control. Clin J Pain. 2008;24(4):291-8.

34. Tsao H, Galea MP, Hodges PW. Reorganization of the cortex is associated with postural control deficits in recurrent low back pain. Brain. 2008;131:2161-71.

35. Ferreira ML, Ferreira PH, Latimer J, Herbert RD, Hodges PW, Jennings MD, et al. Comparison of general exercise, motor control exercise and spinal manipulative therapy for chronic low back pain: A randomized trial. Pain. 2007;131:31-7.

36. França FJR, Burke TN, Claret DC, Marques AP. Estabilização segmentar da coluna lombar nas lombalgias: uma revisão bibliográfica e um programa de exercícios. Fisioter Pesq. 2008;15(2):200-6.

37. Costa LO, Maher CG, Latimer J, Hodges PW, Herbert $\mathrm{RD}$, Refshauge KM, et al. Motor control exercise for chronic low back pain: a randomized placebocontrolled trial. Phys Ther. 2009;89(12):1275-86. 